\title{
Tromboflebitis superficial del pene: primer reporte de caso en el Hospital Universitario Nacional de Colombia y recomendaciones actuales de manejo
}

\section{Superficial Vein Thrombosis of the Penis: A Case Report and Current Recommendations for Diagnosis and Management}

\author{
David Andrés Castañeda ${ }^{1}$ Jonatan Velásquez ${ }^{1}$ Daniel Maya ${ }^{1}$ Julián Pardo ${ }^{1}$ César Capera ${ }^{1}$ \\ Carlos Guzmán ${ }^{1,2}$ Wilfredo Donoso ${ }^{1,2}$ \\ ${ }^{1}$ Unidad de Urología, Departamento de Cirugía; Facultad de \\ Medicina, Universidad Nacional de Colombia, Bogotá, Colombia \\ 2 Unidad de Urología, Hospital Universitario Nacional de Colombia, \\ Bogotá, Colombia \begin{abstract}
residente de Urología, Universidad Nacional de Colombia, Facultad de Medicina - Edificio 471, Oficina 107, Bogotá, Colombia

(e-mail: dacastanedam@unal.edu.co).
\end{abstract} \\ Address for correspondence David Andrés Castañeda, Médico
}

Urol Colomb 2019;28:61-65.

\section{Resumen \\ Palabras Claves \\ - tromboflebitis \\ - enfermedades del pene \\ - pene}

La enfermedad de Mondor es una condición caracterizada por la presencia de tromboflebitis en varios segmentos corporales, fue descrita inicialmente por Henri Mondor en 1939 con descripciones de casos que afectaban la circulación venosa de la reja costal y las glándulas mamarias. Se alude a Braun-Falco en 1955 la primera mención de la trombosis superficial del pene, sin embargo, fue hasta 1958 cuando Helm y Hodge describieron el primer caso con compromiso urogenital masculine. Actualmente se cuenta con información limitada sobre la tromboflebitis superficial del pene (enfermedad peniana de Mondor), por lo tanto, el presente artículo describe el primer caso de tromboflebitis de la vena superficial del pene registrado en el Hospital Universitario Nacional de Colombia y expone una propuesta de abordaje terapéutico actual, basada en una revisión reciente de la literatura.

Superficial vein thrombosis was described by Henri Mondor in 1939. At the start of the experience, the disease affected the venous circulation of the thoracic wall and breasts; Helm and Hodge publicated the first report of penile Mondor's disease in 1958. Currently, there is very little clinical information about penile Mondor's disease. This article shown the first case report of penile Mondor's disease in Colombia and proposes a novel, as well as, current algortihm for management of this disease.

\section{Introducción}

La enfermedad peniana de Mondor fue descrita desde hace más de 50 años ${ }^{1}$; a pesar de contar con registros en la literatura de larga data son pocos los casos reportados actualmente en la literatura mundial. En 2005 Kumar y colaboradores estimaron una incidencia del $1,39 \%{ }^{2}$ Esta enfermedad constituye una de las causas más importantes de dolor peniano en hombres con vida sexual activa; por su curso agudo y la severidad de los síntomas, puede ser una fuente importante de distrés físico y emocional para el hombre que padece la enfermedad. En su received April 26, 2018 accepted July 17, 2018 published online August 15, 2018
DOI https://doi.org/ $10.1055 / \mathrm{s}-0038-1668520$. ISSN 0120-789X. eISSN 2027-0119.
Copyright ( 2019, Sociedad Colombiana License terms de Urología. Publicado por Thieme Revinter Publicações Ltda., Rio de Janeiro, Brazil. Todos los derechos reservados.

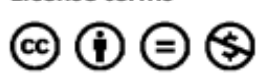


génesis se han postulado factores vinculados a la tríada de Virchow (lesión endotelial, estasis venosa, estado procoagulante) como el coito vigoroso, abstinencia sexual prolongada, aplicación de medicamentos intracavernosos, estados postquirúrgicos, uso de dispositivos externos sexuales, enfermedades de transmisión sexual, uso de sustancias de abuso endovenosas, tumores pélvicos entre otros. $^{3,4}$ Para el momento de elaboración del presente artículo, solo se disponía de 5 referencias clínicas latinoamericanas, ${ }^{5,8,15,21,32}$ una referencia de enfermedad peniana de Mondor en Suramérica ${ }^{7}$ y ninguna referencia en la literatura médica colombiana, por lo tanto, se presenta el primer reporte de caso sobre la tromboflebitis superficial el pene manejado en el Hospital Universitario Nacional de Colombia (Bogotá, Colombia) y se propone un algoritmo clínico para el manejo de esta entidad.

\section{Materiales y Método}

Reporte de caso y revisión de la literatura en las bases de datos Medline, Embase y Lilacs utilizando los términos MeSH: “Trombophlebitis," "Penile disease" y "Penis." Se limitó la búsqueda hacía artículos publicados en los últimos 15 años. Se excluyeron los artículos sin relación a patología peniana. Se obtuvo un total de 16 referencias bibliográficas; algunas referencias adicionales fueron incluidas por su relevancia histórica o clínica.

\section{Reporte de Caso}

Hombre de 47 años, sin antecedentes médicos de relevancia, que consultó al servicio de Urología del Hospital Universitario Nacional de Colombia por dolor peniano de 4 semanas de evolución persistente y que catalogaba en la escala visual análoga con intensidad 4/10; al interrogatorio refería inicio del dolor tras una relación sexual vigorosa. No refirió otros síntomas generales ni del tracto urinario, las erecciones permanecían de buena calidad, sin embargo, éstas empeoraban el dolor. Al examen físico el paciente presentaba un cordón filiforme indurado en la cara dorsal del pene francamente doloroso a la palpación, no se evidenciaron otras lesiones genitourinarias ni estigmas de tromboflebitis en otros segmentos corporales. En el resto del examen físico no se encontraron otros hallazgos patológicos.

El hemograma no presentó alteraciones significativas (Hemoglobina: $14.9 \mathrm{gr} / \mathrm{dL}$, Leucocitos: $7740 / \mathrm{mm}^{3}$, Neutrófilos: 56.5\%, Linfocitos: 36\%, Plaquetas: 312.000/ $\mathrm{mm}^{3}$ ); los tiempos de coagulación se encontraban en límites normales (TP: 11.8 segundos, TPT: 30 segundos, INR: 1.08 ).

La ecografía doppler de pene evidenció la presencia de una imagen hiperecoica de 2 centímetros de longitud y ausencia de flujo sanguíneo en la vena dorsal superficial del pene consistente con trombo intraluminal venoso (-Figs. 1, 2).

El paciente recibió manejo sintomático durante dos semanas (analgésicos vía oral 4 veces al día, antiinflamatorios no esteroideos vía oral 3 veces al día y medidas locales incluyendo heparinoide tópico), se recomendó abstención sexual hasta la resolución de los

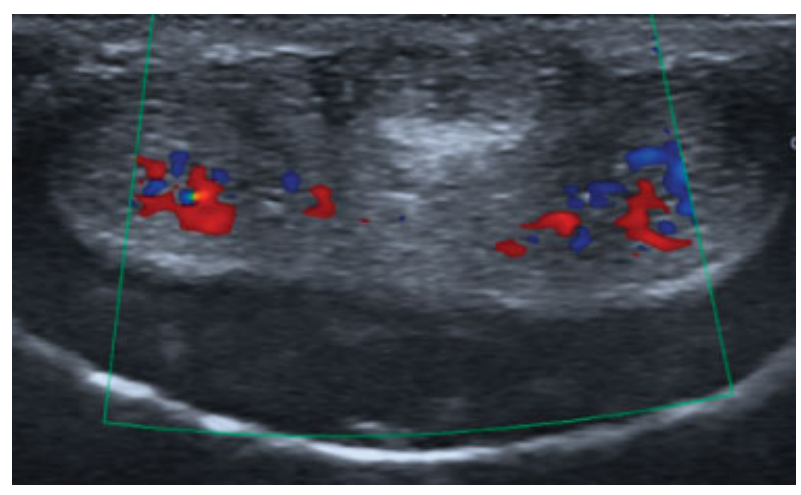

Fig. 1 Corte coronal ecografía doppler de pene con evidencia de ausencia de flujo sanguíneo en la vena dorsal superficial del pene.

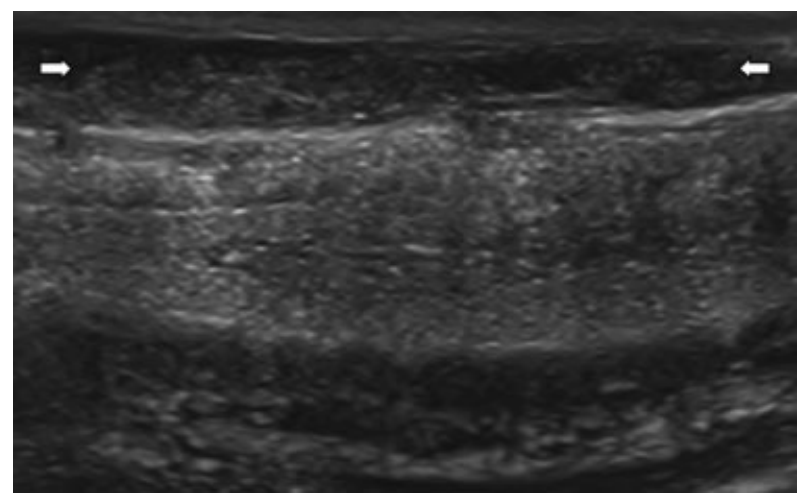

Fig. 2 Corte sagital ecografía doppler de pene que evidencia el trombo intraluminal en la vena dorsal superficial del pene (señalado por las flechas).

síntomas. Luego de dos semanas de manejo médico presentó resolución completa del dolor y de los síntomas locales. Tras el manejo instaurado el paciente retorno a su vida cotidiana sin secuelas funcionales ni deformidad en el pene.

\section{Discusión}

La tromboflebitis de la vena superficial del pene (Enfermedad peniana de Mondor), es una entidad rara y poco frecuente que afecta principalmente a hombres en etapa productiva de la vida (entre los 20 y 57 años). Para el año 2015, no se contaba con más de 50 casos descritos en la literatura mundial ${ }^{6}$; actualmente sólo se dispone de una referencia suramericana ${ }^{7}$ y no hay casos descritos en la literatura médica colombiana. En su patogénesis se ha postulado como eje central factores o condiciones que alteran el balance de la tríada de Virchow (ver - Tabla 1).

Algunos autores han descrito la aparición de la enfermedad peniana de Mondor en situaciones especiales, incluyendo su aparición tras algunos procedimientos quirúrgicos. Arango y col., describieron la tromboflebitis superficial de la vena dorsal del pene como la complicación quirúrgica más frecuente $(2,1 \%)$ en pacientes llevados a varicocelectomía subinguinal ${ }^{8}$; Kutlay y col., ${ }^{9} \mathrm{y}$ Rodríguez y col., ${ }^{10}$ describieron la aparición de la enfermedad peniana de Mondor tras la ejecución de una 
Tabla 1 Factores asociados con la aparición de la Enfermedad de Mondor (todos los factores afectan el balance de la tríada de Virchow - daño endotelial, estasis venosa, estado procoagulante) $3,6,19,21,23$

\begin{tabular}{|c|c|}
\hline $\begin{array}{l}\text { - Coito prolongado } \\
\text { o vigoroso. } \\
\text { - Trauma peniano. } \\
\text { - Abstinencia sexual } \\
\text { prolongada. } \\
\text { - Sobredistensión vesical. } \\
\text { - Uso de dispositivos } \\
\text { vacumm para la } \\
\text { erección. } \\
\text { - Inyección de sustancias } \\
\text { intracavernosas. } \\
\text { - Posoperatorio de } \\
\text { cirugías inguinales, } \\
\text { genitales o pélvicas } \\
\text { (varicocelectomía } \\
\text { subinguinal, herniorrafía } \\
\text { inguinal, entre otras). }\end{array}$ & $\begin{array}{l}\text { - Tumores pélvicos. } \\
\text { - Anemia de células } \\
\text { falciformes. } \\
\text { - Déficit de proteína S y C. } \\
\text { - Déficit de antitrombina III. } \\
\text { - Enfermedad de Behcet. } \\
\text { - Policitemia Vera. } \\
\text { - Tromboflebitis séptica. } \\
\text { - Síndrome paraneoplásico. } \\
\text { - Infección de transmisión } \\
\text { sexual (sífilis, gonorrea). }\end{array}$ \\
\hline
\end{tabular}

herniorrafía inguinal. Zerwerck y col., ${ }^{11}$ describieron la aparición de esa enfermedad tras la ablación láser endovenosa de la vena safena mayor. Day y col., ${ }^{12}$ describieron un caso con aparición de la enfermedad tras un vuelo de larga duración (15 horas).

Shen Hy col., ${ }^{13}$ describieron un caso en el cual se documentó como factor predisponente el aumento de la actividad del factor VIII de la coagulación; Rani ${ }^{14}$ describió un caso de enfermedad concomitante con un episodio de sífilis primaria, sin embargo, es preciso resaltar que los datos descritos por Kumar y col., ${ }^{2}$ demuestran que la prevalencia de enfermedad peniana de Mondor en pacientes con enfermedades de transmisión sexual es baja y sólo 2 de 18 pacientes presentaron tromboflebitis de la vena superficial del pene de forma concomitante con un diagnóstico de enfermedad de transmisión sexual (chancroide y verrugas genitales).

Se han descrito tres fases de la enfermedad: Aguda, que corresponde a la aparición de síntomas intensos en las primeras 24-48 horas; subaguda, que se caracteriza por persistencia ..de los.. síntomas hasta por 8 semanas; tardía, en la que se puede presentar recanalización del lumen de la vena dorsal superficial del pene con reabsorción del coágulo, recanalización vascular y resolución sintomática o persistencia de síntomas por más de 9 semanas. ${ }^{15}$

El diagnóstico de esta entidad se basa en los hallazgos al examen físico (cordón filiforme palpable y doloroso en la cara dorsal del pene); por años se ha postulado el uso de imágenes diagnósticas (Ecografía doppler de pene, Resonancia magnética nuclear de pene) como ayudas adecuadas para el abordaje diagnóstico $^{16}$ dados los hallazgos confirmatorios que se pueden evidenciar (ausencia de flujo venoso, edema circundante a vena dorsal superficial del pene, lesión hiperecoica no compresible en el trayecto de la vena dorsal del pene, flujo de alta resistencia en la arteria cavernosa ${ }^{17,18}$ ), sin embargo, recientemente Polat y col., ${ }^{19}$ han puesto de manifiesto la necesidad de disminuir el uso del armamentario imagenológico en una entidad cuya historia natural está dirigida hacia la autorresolución. Es preciso aclarar que dicha experiencia utilizó la ecografía doppler con aplicación intracavernosa de agentes vasoactivos para la ejecución del diagnóstico, sin embargo, los autores consideramos, de forma similar a los descrito por Yeo Han y col., en su experiencia, ${ }^{20}$ que el uso de agentes vasoactivos no resulta imprescindible para el abordaje diagnóstico de esa entidad y que la ecografía doppler de pene sin sustancias vasoactivas puede ser un recurso útil que genera datos suficientes para el abordaje diagnóstico de la enfermedad peneana de Mondor.

Dentro de los diagnósticos diferenciales que el urólogo debe excluir en pacientes con lesiones filiformes induradas, palpables y dolorosas en el pene se encuentra la linfangitis esclerosante del pene. ${ }^{21,22}$ En los últimos años se ha logrado definir que la presencia de una lesión filiforme longitudinal palpable, flujo de alta resistencia en la arteria cavernosa del pene en la ecografía doppler o cambios en ecográficos en la vena dorsal del pene (ausencia de flujo, ocupación por imagen hiperecogénica o imposibilidad para lograr la compresión con el ecógrafo) apoyan el diagnóstico clínico de enfermedad peniana de Mondor. ${ }^{23,24}$

Los hallazgos histopatológicos característicos y diferenciales de la enfermedad de Mondor son: edema de las células endoteliales, aumento de tejido conectivo en las paredes vasculares, tromboflebitis obliterante, infiltración de las áreas perivasculares por células de línea blanca (linfocitos, histiocitos y plasmocitos) y marcación positiva del endotelio con anticuerpos monoclonales para CD31, CD34 y vWF (Factor de Von Willebrand). ${ }^{25,26}$

El manejo de la enfermedad de Mondor se basa en el control sintomático; el uso de analgésicos, antiinflamatorios, heparinoides tópicos junto a la abstinencia sexual (coito y masturbación) durante la fase aguda y subaguda constituyen el pilar terapéutico. ${ }^{27,28}$ Existen estudios que revelan una tasa de éxito de hasta el $92 \%$ en pacientes llevados a manejo conservador. $^{29}$ Algunos autores han descrito buenos resultados en el control sintomático de la enfermedad con el uso de ácido acetil salicílico ${ }^{30}$ y corticoides tópicos ${ }^{31} \mathrm{u}$ orales. ${ }^{10}$ Se debe considerar el manejo quirúrgico mediante trombectomía o resección quirúrgica de la vena dorsal superficial del pene si los síntomas no modulan con el manejo médico o si persisten más allá de las 9 semanas de iniciado el cuadro clínico. ${ }^{32,33}$ Proponemos un algoritmo de manejo práctico basado en las experiencias descritas en la literatura mundial (-Fig. $\mathbf{3}$ ).

Existen pocos datos clínicos sobre el pronóstico o secuelas a mediano o largo plazo en pacientes con tromboflebitis superficial de la vena dorsal del pene; por años se ha considerado a la enfermedad peniana de Mondor como una enfermedad de carácter benigno con tendencia a la autorresolución. Recientemente Ozkan y col., lograron definir en su serie de casos (30 pacientes) que la enfermedad de Mondor no altera la función eréctil y ni genera deformidad peniana persistente ${ }^{34}$ tras dos meses de seguimiento clínico.

El caso expuesto tuvo como detonante de la enfermedad un coito vigoroso, a la ecografía doppler de pene presentó los signos radiológicos clásicos para enfermedad peniana de Mondor, recibió manejo médico en la fase aguda - subaguda 


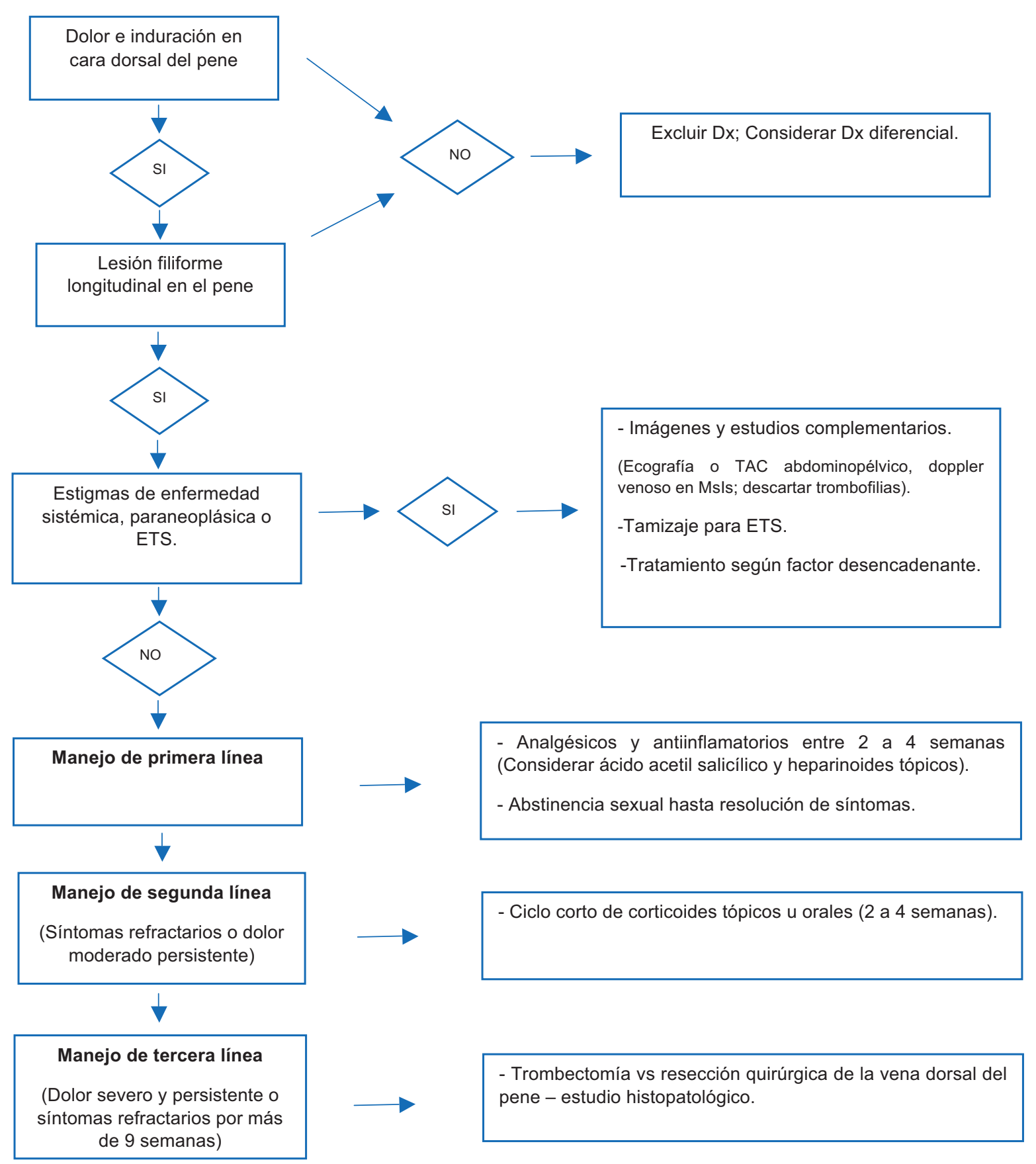

Fig. 3 Propuesta de algoritmo de manejo para la enfermedad peniana de Mondor (Fuente: El autor). ETS: Enfermedad de transmisión sexual; TAC: Tomografía axial computarizada; Dx: diagnóstico; Msls: Miembros inferiores.

de la enfermedad y de forma similar a las experiencias descritas en la literatura mundial alcanzó un desenlace clínico favorable ya que presentó resolución sintomática tras dos semanas de manejo conservador y en el seguimiento clínico ejecutado tras un mes de ocurrido el evento agudo no presentó alteraciones anatómicas o funcionales significativas.

\section{Conclusiones}

La enfermedad peniana de Mondor consiste en la trombosis de las venas del circuito superficial del pene, es una entidad poco frecuente cuya aparición está determinada por factores que afectan el balance de la tríada de Virchow. El manejo médico es el estándar de tratamiento actual y ha demostrado altas tasas de curación y control sintomático; el manejo quirúrgico debe reservarse sólo para casos refractarios o con síntomas de difícil control que afecten de forma significativa la calidad de vida del paciente. El caso presentado constituye la primera experiencia clínica reportada en Colombia, y de forma similar a lo descrito en la literatura mundial, presentó una adecuada evolución clínica tras recibir manejo médico.

Financiación

Ninguno declarado por los autores. 
Conflicto de Interés

Ninguno declarado por los autores.

Agradecimientos

A Camila Ruiz Tacha por su apoyo en la revisión de estilo del presente artículo.

\section{Bibliografía}

1 Helm JD Jr, Hodge IG. Thrombophlebitis of a dorsal vein of the penis; report of a case treated by phenylbutazone (butazolidin). J Urol 1958;79(02):306-307

2 Kumar B, Narang T, Radotra BD, Gupta S. Mondor's disease of penis: a forgotten disease. Sex Transm Infect 2005;81(06):480-482

3 Laroche JP, Galanaud J, Labau D, et al. Mondor's disease: what's new since 1939? Thromb Res 2012;130(Suppl 1):S56-S58

4 Rowe M, Depraetere K. A presentation of penile Mondor's disease. Int J STD AIDS 2012;23(09):681-682

5 Molina Escudero R, Cabello Benavente R, Monzó Gardiner JI, et al. Síndrome de Mondor. Revisión de la literatura. A propósito de un caso. Arch Esp Urol 2009;62(04):317-319

6 Manimala NJ, Parker J. Evaluation and treatment of penile thrmbophlebitis (Mondor's Disease). Curr Urol Rep 2015;16(06):39

7 Martucci R, Zerati M, Muller M. Thrombosis of the superficial dorsal penile vein (penile mondor's disease).J Bras Urol 1999;25(01):93-94

8 Arango O, Lorente JA, Nohales G, Rijo E, Bielsa O. Superficial dorsal penile vein thrombosis: a little-known complication of subinguinal varicocelectomy. BJU Int 2011;107(01):95-98

9 Kutlay J, Genc V, Ensari C. Penile Mondor's disease. Hernia 2008; 12(05):557-558

10 Rodríguez T, Valdés B, González M, et al. Tromboflebitis de Mondor del pene tras hernioplastia inguinal. Rev Cuba Med Mil 2014;43(03):386-393

11 Zerweck C, Knittel M, Zeller T, Schwarz T. Penile Mondor's syndrome after endovenous treatment of the great saphenous vein with $1470 \mathrm{~nm}$ diode laser. Phlebology 2015;30(03):217-219

12 Day S, Bingham JS. Mondor's disease of the penis following a longhaul flight. Int J STD AIDS 2005;16(07):510-511

13 Shen HL, Liu SP, Wang SM, Tsay W, Hsieh JT. Elevated plasma factor VIII coagulant activity presenting with thrombophlebitis of the deep dorsal vein of the penis. Int J Urol 2007;14(07):663-664

14 Rani R. Mondor's disease of the penis associated with primary syphilis. Int J STD AIDS 2009;20(07):510-511

15 Aguilar-García JJ, Domínguez-Pérez AD, Iribarren-Marín MA, Talegón-Meléndez A. Enfermedad de Mondor. . Una forma inusual de trombosis venosa superficial. Rev Clin Esp 2011;211(04):216-217

16 Kantarcı UH, Dirik A, Öztürk YE, Kiriş İ, Duymuş M. Doppler ultrasound and magnetic resonance imaging findings of penile Mondor's disease: Case report and literature review. Pol J Radiol 2016;81:36-38

17 Dell'Atti L. Role of ultrasonography with color-Doppler in diagnosis of penile Mondor's disease. J Ultrasound 2013;17(03):239-241
18 Machan K, Rojo-Carmona LE, Marquez-Moreno AJ, et al. Ultrasound diagnosis of three cases of Mondor's disease. Arch Esp Urol 2012;65(02):262-266

19 Polat H, Yucel M, Gok Aet al. Penile Mondoŕs Disease: Primum non nocere!. Urol J 2015;12(02):2096-2098

20 Han HY, Chung DJ, Kim KW, Hwang CM. Pulsed and color Doppler sonographic findings of penile Mondor's disease. Korean J Radiol 2008;9(02):179-181

21 Rosety J, García-Baquero R, Conde A, et al. Trombosis aislada de la vena superficial dorsal del pene - Flebitis de Mondor. Presentación de un caso y revisión de la bibliografía. Rev Int Androl 2011;9(04):174-176

22 Rivero Rodríguez W, Pradilla Valbuena J. Lifangitis esclerosante no venérea del pene. Presentación de 2 casos y revisión de la literatura. Urol Colomb. 2017http://dx.doi.org/10.1016/j.uroco.2017.04.006

23 Ramos Barseló E, Portillo Martín JA, Correas Gómez M, et al. Enfermedad de Mondor versus linfangitis esclerosante de pene. Arch Esp Urol 2008;61(07):837-840

24 Ozturk H. Penile Mondoŕs Disease. Basic and clinical andrology 2014;24:5

25 Alvarez-Garrido H, Sanz-Muñoz C, Pericet-Fernández L, GarridoRíos AA, Miranda-Romero A. Immunohistochemical clues to the diagnosis of Mondor's disease of the penis. Clin Exp Dermatol 2009;34(08):e663-e665

26 Ichinose A, Fukunaga A, Terashi $\mathrm{H}$, et al. Objective recognition of vascular lesions in Mondor's disease by immunohistochemistry. J Eur Acad Dermatol Venereol 2008;22(02):168-173

27 Walsh JC, Poimboeuf S, Garvin DS. A common presentation to an uncommon disease. Penile Mondor's disease: a case report and literature review. Int Med Case Rep J 2014;7:155-157

28 Tiplica GS, Sălăvăstru CM, Nedelcu L. Mondor's phlebitis of the penis: an investigational periplus. J Eur Acad Dermatol Venereol 2012;26(03):393-394

29 Al-Mwalad M, Loertzer H, Wicht A, Fornara P. Subcutaneous penile vein thrombosis (Penile Mondor's Disease): pathogenesis, diagnosis, and therapy. Urology 2006;67(03):586-588

30 Davarci M, Gvuven E, Gokce A, et al. Penile Mondor's disease can be effectively treated with the use of an acetyl salicylic acid and pentoxifylline combination. Turk J Med Sci 2010;40(05): 735-738

31 Yang Y, Qian SQ, Wei Q, Yuan JH. Treat penile Mondor's disease with corticosteroid cream. Kaohsiung J Med Sci 2017;33(08): 424-425

32 Rodríguez Faba O, Parra Muntaner L, Gómez Cisneros SC, Martín Benito JL, Escaf Barmadah S. Trombosis de la vena dorsal del pene (Flebitis de Mondor). Aportación de un nuevo caso. Actas Urol Esp 2006;30(01):80-82

33 Sasso F, Gulino G, Basar M, Carbone A, Torricelli P, Alcini E. Penile Mondors' disease: an underestimated pathology. Br J Urol 1996; 77(05):729-732

34 Özkan B, Coskuner ER, Turk A, Akkus E, Yalçın V. Penile Mondor disease and its effect on erectile function: results of 30 patients. Urology 2015;85(01):113-117 